\title{
Ten-year follow-up of a randomized controlled clinical trial in chronic hepatitis delta
}

\author{
Anika Wranke $^{1}$ (D) | Svenja Hardtke ${ }^{1,2}$ | Benjamin Heidrich ${ }^{1,2}$ | George Dalekos ${ }^{3}$ | \\ Kendal Yalçin ${ }^{4}$ | Fehmi Tabak $^{5}$ | Selim Gürel ${ }^{6}$ | Yilmaz Çakaloğlu ${ }^{7}$ Ulus S Akarca ${ }^{8}$ | \\ Frank Lammert $^{9}$ | Dieter Häussinger ${ }^{10}$ | Tobias Müller ${ }^{11}$ | Michael Wöbse ${ }^{1}$ | \\ Michael P. Manns ${ }^{1,2}$ | Ramazan Idilman ${ }^{12}$ | Markus Cornberg ${ }^{1,2}$ (D) \\ Heiner Wedemeyer ${ }^{2,13}$ | Cihan Yurdaydin ${ }^{12,14}$ (i) \\ ${ }^{1}$ Department of Gastroenterology, Hepatology and Endocrinology, Hannover Medical School, Hannover, Germany \\ ${ }^{2}$ German Center for Infection Research (DZIF), Partner Site HepNet Study-House, Hannover, Germany \\ ${ }^{3}$ Department of Medicine and Research Laboratory of Internal Medicine, University Hospital of Larissa, Larissa, Greece \\ ${ }^{4}$ Dicle University Medical Faculty, Diyarbakir, Turkey \\ ${ }^{5}$ Department of Infectious Diseases Cerrahpaşa School of Medicine, Istanbul University, Istanbul, Turkey \\ ${ }^{6}$ Uludağ University Medical School, Bursa, Turkey \\ ${ }^{7}$ Memorial Hospital, Istanbul, Turkey \\ ${ }^{8}$ Ege University Medical Faculty, Izmir, Turkey \\ ${ }^{9}$ Department of Medicine II, Saarland University Medical Center, Saarland University, Homburg, Germany \\ ${ }^{10}$ Department of Gastroenterology, Hepatology and Infectious Diseases, Heinrich Heine University, Düsseldorf, Germany \\ ${ }^{11}$ Charite University, Berlin, Germany \\ ${ }^{12}$ Department of Gastroenterology, Ankara University School of Medicine, Ankara, Turkey \\ ${ }^{13}$ Department of Gastroenterology and Hepatology, Essen University Hospital, Medical Faculty of the University Duisburg-Essen, Essen, Germany \\ ${ }^{14}$ Department of Gastroenterology and Hepatology, Koç University Medical School, Istanbul, Turkey
}

\section{Correspondence}

Svenja Hardtke, Department for

Gastroenterology, Hepatology and

Endocrinology, Hannover Medical School,

30625 Hannover.

Email: Hardtke.svenja@mh-hannover.de

Cihan Yurdaydin, Department of Gastroenterology and Hepatology, Koç University Medical School, Davutpaşa

Caddesi No: 4, Topkapi, 34010 Istanbul.

Email: cyurdaydin@ku.edu.tr and cihan.

yurdaydin@medicine.ankara.edu.tr

Funding information

This study was funded by the German Centre for Infection Research (DZIF), partner site Hannover-Braunschweig with a grand to the HepNet Study-House, the German Liver Foundation and an EASL registry grant.

\begin{abstract}
Hepatitis delta virus (HDV) infection causes the most severe form of viral hepatitis. PEG-interferon alpha-2a (PEG-IFN $\alpha-2 a$ ) is the only effective treatment but its longterm clinical impact is unclear. The aim of this study was to investigate the long-term outcome after 48 weeks of pegylated interferon alpha-2a therapy. We performed a retrospective follow-up study of the Hep-Net-International-Delta-HepatitisIntervention-Study 1 (HIDIT-I trial). Patients had received 48 weeks of treatment with either PEG-IFN $\alpha$-2a plus adefovir dipivoxil (ADV) (Group I), PEG-IFN $\alpha$-2a alone (Group II) or adefovir dipivoxil alone (Group III). Liver-related complications were defined as liver-related death, liver transplantation, liver cancer and hepatic decompensation defined as development of Child-Pugh scores B or C or an increase in Model for End-stage Liver Disease (MELD) scores of five or more points in relation to baseline values. Patients were considered for further analysis when they were retreated
\end{abstract}


with PEG-IFN $\alpha-2 a$. Follow-up data (at least 1 visit beyond post-treatment week 24) were available for 60 patients [Group I, $(n=19)$, Group II $(n=20)$, Group III $(n=21)$ ]. Mean time of follow-up was $8.9(1.6$ - 13.4) years. 19 patients were retreated with IFN-based therapy: $42 \%(n=8)$ in PEG-IFN $\alpha-2 a$ arms and $58 \%(n=11)$ in the adefovir only arm. Clinical complications on long-term follow-up occurred in 17 patients and were associated with nonresponse to therapy and baseline cirrhosis. The annual event-free survival rate in patients with cirrhosis vs noncirrhotic patients at year 5 and 10 was $70 \%$ vs $91 \%$ and $35 \%$ vs $76 \%$. Long-term follow-up of a large randomized clinical trial suggests that off-treatment HDV RNA response to PEG-IFN $\alpha-2 a$ treatment leads to improved clinical long-term outcome.

KEYWORDS

chronic hepatitis, clinical outcome, delta virus, endpoint, Hepatitis D

\section{1 | INTRODUCTION}

The hepatitis delta virus (HDV) is the smallest RNA-virus, which requires the hepatitis B surface antigen (HBsAg) for viral assembly. HDVinduced acute or chronic hepatitis can therefore occur only as either a coinfection or superinfection with the hepatitis $B$ virus (HBV). ${ }^{1-3}$ In chronic hepatitis delta (CHD), HDV is the dominant virus in the majority of patients although a dynamic shift of the dominant virus over time is possible. ${ }^{4,5}$ Infections caused by HDV are the most severe form of viral hepatitis, frequently leading to cirrhosis and consecutive liver-related complications, including hepatocellular carcinoma (HCC) and liver-related death. ${ }^{1,6}$ Eight different genotypes of HDV have been described, which may have an impact on disease course or response to interferon therapy. ${ }^{2,7}$ On a global scale, the most prevalent HDV genotype is genotype 1. In Germany, Greece and Turkey where this study was conducted, the HDV genotype was exclusively genotype 1. ${ }^{2,8}$ Although hepatitis delta is an orphan disease, it is a major health burden in some regions. Globally, 292 million individuals are hepatitis B surface antigen positive; of them approximately $5 \%$ are coinfected with $\mathrm{HDV} .{ }^{9,10}$ Further, a recent study suggested that this may even be an underestimation although serious critique related to several methodological problems have been raised. ${ }^{11-13}$

Treatment with pegylated interferon alpha (PEG-IFN $\alpha$ ) is still the only available therapy with proven efficacy. Studies showed variable virological response rates of $17 \%-47 \% .{ }^{14-18}$ One of the largest randomized treatment trial, the Hep-Net-International-Delta-HepatitisIntervention-Study 1 (HIDIT-I), investigated the efficacy of 48 weeks of PEG-IFN $\alpha$-2a plus adefovir as compared with either drug alone. ${ }^{15}$ The primary endpoint of that study was HDV RNA negativity and normalization of alanine aminotransferase (ALT) at end of therapy (EOT) which was achieved in $24 \%$ of the patients. In a previous study, we have reported the five-year follow-up data of the HIDIT-I study. The most important finding of this latter study was that late relapse, defined as detectable HDV RNA of patients with undetectable HDV RNA at 24 weeks follow-up occurred in $56 \%$ of patients. ${ }^{19}$ However, none of the patients, who tested negative at follow-up week 24 , had developed a liver-related clinical endpoint during 5 years of post-treatment follow-up. We have previously reported clinical long-term course after PEG-IFN $\alpha$-2a based therapies ${ }^{17,20,21}$ but these were retrospective single-centre studies based on databases of the respective centres.

The aim of this study was to investigate the long-term outcome after a 48 weeks course of PEG-IFN $\alpha$-2a therapy. The importance of this study is that for the first time a 10-year long-term outcome data of a randomized controlled multicentre study is performed.

\section{2 | METHODS}

\section{1 | Study population}

We here performed a retrospective long-term follow-up study of the patients included in the HIDIT-I trial. ${ }^{15}$ Initially, 90 chronic hepatitis delta patients were included in the study. All patients were treated for 48 weeks with the assigned medications. The first treatment arm (Group I: $\mathrm{n}=31$ ) received PEG-IFN $\alpha$-2a plus adefovir ADV, the second arm (Group II: $\mathrm{n}=29$ ) received PEG-IFN $\alpha$-2a plus placebo, and the third group (Group III: $n=30$ ) received ADV alone. Ten patients withdrew. Altogether, 80 patients completed the study. 77 patients completed post-treatment week 24 follow-up (Group I: 24, Group II: 25, Group III: 28). Long-term follow-up data of at least one year after end of treatment (EOT) were available from 60 patients (Group I: 19, Group II: 20, Group III: 21) with a mean follow-up period of 8.9 (1.6-13.4) years (Figure 1). From 18 patients, the 10-year FU data were available. In this cohort, some patients were lost to follow-up or developed a clinical endpoint.

\section{2 | Data collection}

Data were collected every year for 10 years. Patients $(n=60)$ were followed until they reached a clinical endpoint or got lost to follow-up. Patients who based on the treating physician's discretion 
FIGURE 1 Number of patients in the treatment arms and the follow-up study

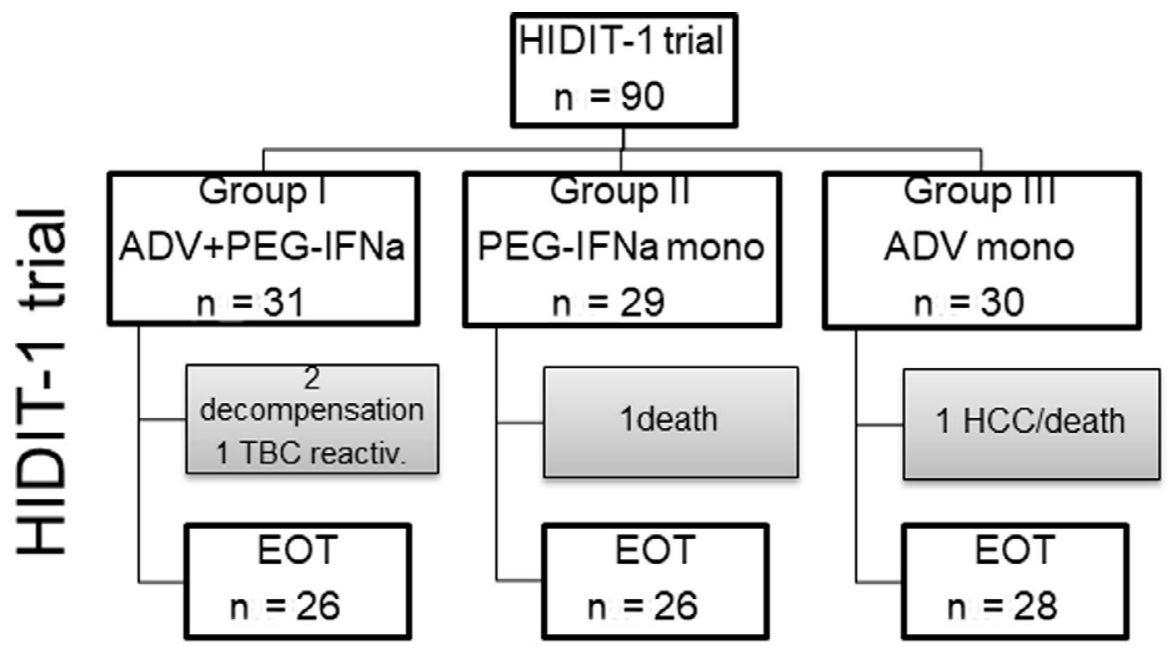

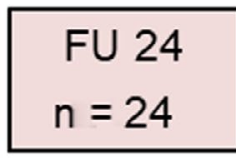
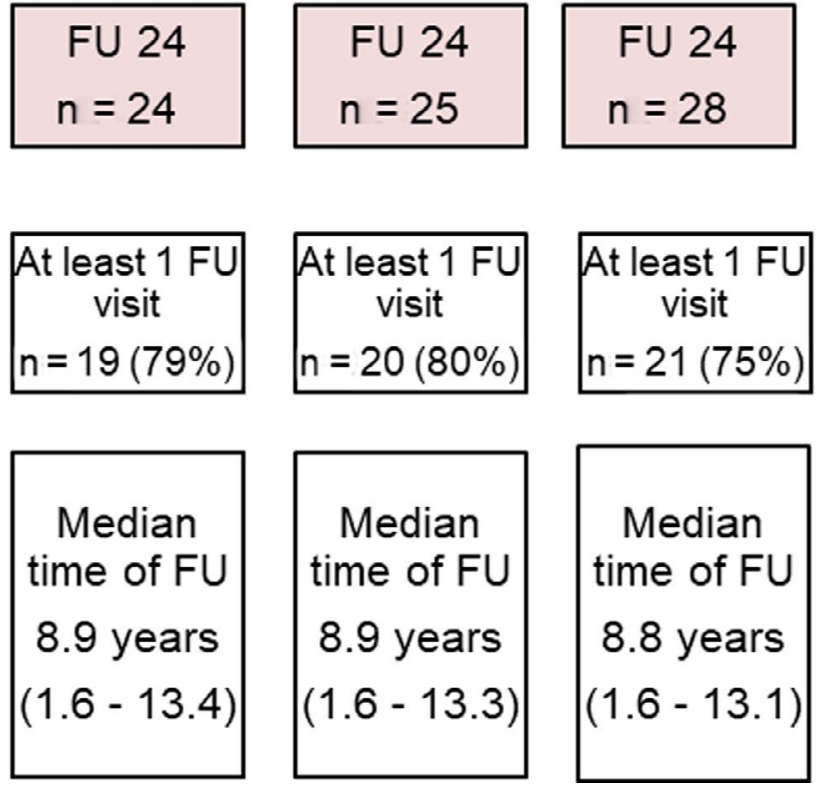

received a second course of IFN were included in the long-term follow-up. During routine visits biochemical (ALT, AST, GGT, ALP, bilirubin, creatinine and albumin), haematological (platelet counts and prothrombin time) and virological parameters (HDV RNA and HBV DNA) were locally measured. For the initial study, a quantitative HDV RNA assay was tested centrally at Hannover Medical School. ${ }^{2}$ During the long-term follow-up study, we relied on locally measured HBV DNA and HDV RNA. For HBV DNA, commercially available tests such as the Roche Cobas TaqMan assay or other highly sensitive assays were used. HDV RNA measurements were performed with in-house real time PCR assays. Quantitative HBsAg was measured with the Abbott Architect assay. ${ }^{22}$

\section{3 | Definition of clinical and virological endpoints}

Clinical endpoints were defined as death, and liver transplantation and hepatic decompensation defined as development of Child-Pugh scores $\mathrm{B}$ or $\mathrm{C}$ or an increase in Model for End-stage Liver Disease (MELD) scores of five or more points in relation to baseline values or development of HCC.

Several virological endpoints were assessed: undetectable HDV RNA at last available visit denotes HDV RNA negativity either at the end of long-term follow-up (LT FU) or the last visit before a patient is lost on follow-up. Negative HDV RNA at any time point denotes patients who were HDV RNA negative at least once during long-term follow-up. Late-HDV RNA relapse is defined as HDV RNA positivity at least once after follow-up 24 week response. Maintained virological response (MVR) was defined as undetectable HDV RNA for at least 2 years during follow-up.

\subsection{Statistics}

Statistical analyses were performed by using SPSS software (version 25) (SPSS Inc). 
All parameters were described as mean \pm SD. $P$ values $<.05$ were considered as statistically significant. Continuous parametric variables were analysed by t tests. For nonparametric parameters, MannWhitney $U$ tests were used. A chi-square test was calculated for the comparison of discrete variables. In case of an expected cell count $<5$, Fisher's exact test was used instead. Parameters that were associated with a better clinical long-term outcome in univariate logistic regression models $(P<.05)$ were additionally compared in multivariate analysis. Multivariate logistic regression analyses were performed by using the likelihood ratio test for backward selection. Odds ratios (ORs), including their 95\% confidence intervals ( $\mathrm{Cls}$ ), were calculated for the logistic regression models. The association of parameters with clinical long-term outcome was also calculated in a time-depending Cox-regression model, in which case hazard ratios (HRs) were calculated. Using Kaplan-Meier analysis, we estimated the cumulative event-free survival within various groups of therapy. In these groups, significant differences concerning event-free survival were indicated by log-rank tests. Disease-free survival (DFS) was estimated using the Kaplan-Meier method for the entire study cohort or subgroup and then compared using univariate log-rank tests between groups. Besides, points were allocated in relation to the logistic regression models. For significant parameters for HBsAg loss, cut-offs were built using ROC curves with the help of the Youden index. For the calculated cut-offs, sensitivity, specificity and positive as well as negative predictive values (PPV, NPV) were calculated by the use of $2 \times 2$ tables.

\subsection{Ethics}

The HIDIT-I trial was approved by the Ethics Committees of each participating institution in line with the 1975 Declaration of Helsinki. Each patient signed a written informed consent form. The following studies concerning the follow-up used information collected during routine visits. Retrospective analyses were approved by the central coordinating Ethics Committee of Hannover Medical School (No 3388).

\section{3 | RESULTS}

\section{1 | Patients}

The mean time of follow-up was 8.9 (1.6 - 13.4) years (Figure 1). 58 out of the included 60 patients were part of the 5 -year follow-up analysis of Heidrich et al. ${ }^{19}$

Baseline characteristics were described in Table 1 and did not differ from the HIDIT-1 ${ }^{15}$ or the 5 year follow-up population (data not shown). ${ }^{19}$

\subsection{Outcome}

The long-term follow-up study focused on virological response and the development of liver-related clinical endpoints.
TABLE 1 Baseline characteristics

\begin{tabular}{|ll} 
& $\begin{array}{l}\text { Patients in the } \\
\text { follow-up study }(\mathbf{n}=60)\end{array}$ \\
\hline Male sex & $35(58.3 \%)$ \\
\hline Age, mean \pm SD range & $39.59 \pm 11.0$ \\
& $(17.6-62.64)$
\end{tabular}

\subsection{1 | Virological response}

The analysis of virological response was carried out in 60 patients with long-term follow-up. Undetectable HDV RNA at follow-up 24 was described in 14 patients (seven each of groups I and II, respectively). Of the 14 patients, 6 had a maintained virological response. In the rest of 8 patients, late-HDV RNA relapses occurred (Figure 2A), between years 2 and 9 (Table S1a). Of these 8 patients, five were retreated with IFN-based therapy but this led to HDV RNA negativity during the entire follow-up in only one patient.

Relapse was associated with a higher baseline HDV RNA $(P=.02)$ but not with quantitative HDV RNA levels at week $24(P=.34)$ or week 48 ( $P=$.15) (Table S2). Further, relapse was unaffected by type of IFN-based therapy, gender, age, HBV DNA or log qHBsAg levels at baseline, on-treatment week 24 , week 48 , EOT and follow-up week 24 (data not shown).

Overall, 22 patients were HDV RNA negative at the last available visit. Of these 22 patients, six were negative throughout the follow-up period as mentioned above; seven patients became negative after IFN retreatment and stayed negative during the whole longterm follow-up (one in the PEG-IFN $\alpha-2 a$ arms, six in the ADV arm) (Figure S3). Two patients had fluctuating HDV RNA after retreatment and seven patients became negative after the HIDIT-I-based therapy between follow-up year 1 to 10 (Figure S3, Table S1a,b). Clinical, biochemical and demographic data of these latter seven late responding patients are shown in Table S4. Only one of these patients developed a liver-related event. Comparing these late responding patients to patient without response, there were no differences concerning HDV RNA at different time points, but late responding patients had statistically lower HBsAg levels at baseline, week 24 and 48 (Table S5). Biochemical and haematological (ALT, AST, gamma-GT, bilirubin, platelets) parameters were not associated with HDV RNA response. Comparing early vs late viral responder patients, patients who respond during therapy had significantly lower 

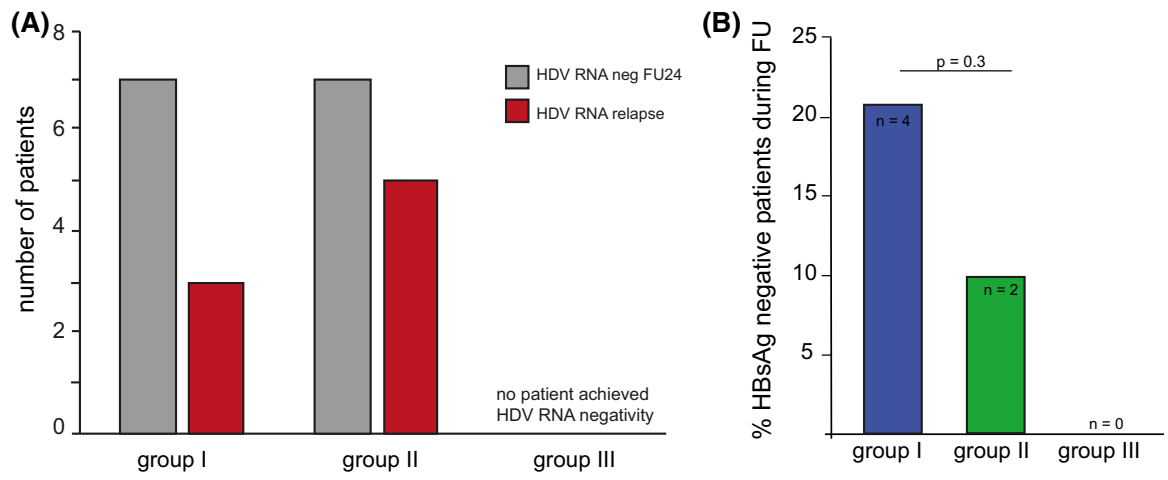

FIGURE 2 A, Number of patients with HDV RNA follow-up 24 response (FU24) and HDV RNA relapses during long-term follow-up (10 y) grouped by the different treatment arms (I ADV + PEG-IFN $\alpha-2 a$; II PEG-IFN $\alpha-2$ a mono and III ADV mono). B, Percent of patients with negative HBsAg during LTFU (10 y) grouped by the different treatment arms. No patient treated with ADV monotherapy experienced HBsAg clearance $(P=.04)$

levels of HDV RNA levels at baseline, week 48 and 72, but no difference in HBsAg levels was observed (Table S6).

Retreatment was not associated with negative HDV RNA at the last available visit $(P=.3)$. The initial HIDIT-I therapy with IFN was also not associated with negative HDV RNA at the last available visit $(P=.16)$. During the whole long-term follow-up, 37 patients became HDV RNA negative at least one time, which is named as negative HDV RNA at any time point. Negative HDV RNA at any time of the follow-up was neither associated with the HIDIT-1 therapy $(P=.38)$ nor with IFN retreatment $(P=.21)$. Negative HDV RNA at any time point was associated with negative $\mathrm{HBsAg}(P=.04)$. The virological changes of patients with EOT undetectable HDV RNA or a $>2$ or $>1$ log decline of HDV RNA at EOT compared to baseline, respectively, are given in Table S7.

\subsection{2 | HBsAg}

Nine patients cleared HBsAg. Of these nine patients, two patients were already $\mathrm{HBs} \mathrm{Ag}$ negative and had developed anti $\mathrm{HBs}$ at week 72. Interestingly, one of them became $\mathrm{HBsAg}$ positive at his last visit. The remaining seven patients cleared HBsAg during longterm follow-up, of whom one did so after hepatic decompensation and 2 after retreatment with PEG-IFN $\alpha-2 a$. Thus, these three patients were not considered in the long-term follow-up analysis since in one patient HBsAg clearance had occurred after a predefined endpoint (hepatic decompensation) and in the other two patients $\mathrm{HBsAg}$ clearance may have been induced by IFN retreatment (Figure 2B). All HBsAg negative patients were also HDV RNA negative (Table S1 a and b). There were no significant differences between the two PEG-IFN $\alpha$-2a treatment arms in the context of HBsAg clearance $(P=.3)$, but no patient treated with ADV monotherapy experienced HBsAg clearance ( $P=.04$ vs PEG-IFN $\alpha-2 a$ treated patients).

Predictive factors for negative HBsAg were as follows: log HDV RNA at week $24(P<.01)$, negative HDV RNA at week $24(P=.02)$, and log HBsAg at baseline, week 24, 48 and 72 (all $P<.01$ ). For these parameters, receiver operating characteristic (ROC) curve analysis was performed and the following cut-offs built using the Youden index: $\log \mathrm{HBsAg}(\mathrm{IU} / \mathrm{mL}$ ) at baseline (cut-off 3.5), week 24 (cut-off 3.2), 48 (cut-off 3.2) and 72 (cut-off 1.5): log HDV RNA (IU/mL) at week 24 (cut-off 1.1). The AUROC for predicting HBsAg loss at baseline, week 24, week 48 and week $72 \mathrm{HBsAg}$ were 0.93, 0.84, 0.90 and 0.83 , respectively. Based on the cut-offs mentioned above, sensitivity, specificity, PPV and NPV were calculated considering $\mathrm{HBsAg}$ loss as the value to be tested (Table S8).

\subsection{3 | Clinical long-term outcome}

During treatment, clinical events occurred in four patients; two developed hepatic decompensation and two died. ${ }^{15}$ During long-term follow-up of patients without IFN retreatment, a total of 12 patients (12/60; 20\%) developed liver-related clinical endpoints. The first appearing clinical endpoints were as follows: 10 had hepatic decompensation, and two had HCC. Of the 10 patients with initial hepatic decompensation, 4 developed HCC on top and 2 patients also decompensated after development of HCC. Six patients underwent liver transplantation, three due to HCC. Overall 4 patients died of liver-related causes (two due to HCC one of whom was transplanted, one because of variceal bleeding and one from an acute liver decompensation with a Child-Pugh $\mathrm{C}$ liver cirrhosis). The incidence of decompensation was 3.126 per 100 person-years, of HCC 1.25 per 100 person-years, of liver transplantation 1.876/100 people-year and of death $1.246 / 100$ people-year.

In patients who received IFN retreatment, 3 more patients decompensated and two additional patients died, from nonliver-related causes one secondary to superior mesenteric artery thrombosis and the other from complication of brain surgery for multiple brain meningiomas.

Endpoints developed after a mean period of 5.9 (1.6-13.4) years (Figure 3B). The cumulative clinical event-free survival in patients without IFN retreatment at years 1, 5 and 10 was $94 \%, 87 \%$ and $60 \%$, respectively. 
(A)

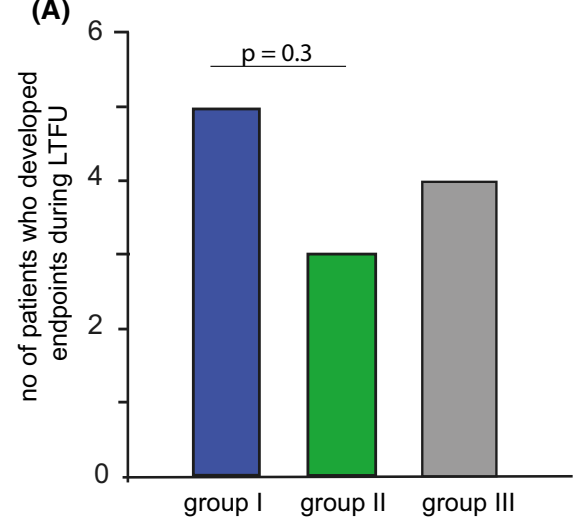

(B)

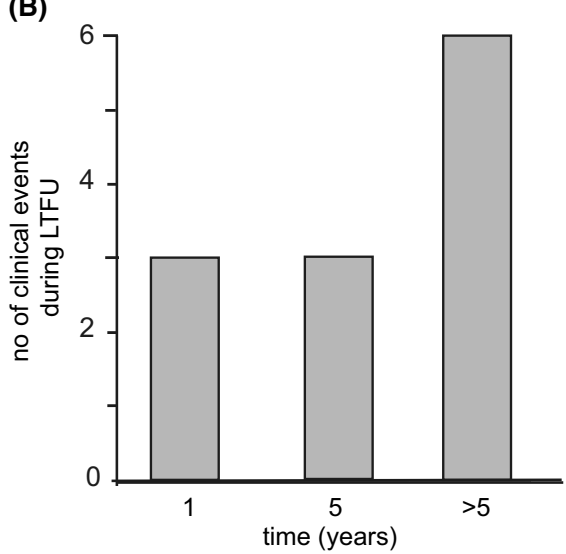

FIGURE 3 A, Number of patients who developed clinical endpoints (defined as: death, liver transplantation and hepatic decompensation defined as development of Child-Pugh scores B or C or an increase in Model for End-stage Liver Disease (MELD) scores of five or more points in relation to baseline values or development of $\mathrm{HCC}$ ) during long-term follow-up grouped by the different treatment arms (I ADV + PEG-IFN $\alpha$-2a; II PEG-IFN $\alpha$-2a mono and III ADV mono). B, Number of clinical events during the follow-up period

No significant differences in clinical outcome could be observed between the three treatment arms in Kaplan-Meier analyses $(P=.3-$ $P=.5$ ) or between the ADV and the combined PEG-IFN $\alpha-2$ a groups $(I+I I)(P=.6)$ (Figure 4A,B).

Further, there was no difference between those who received retreatment and those not retreated in terms of clinical event development $(P=.70)$.

Patients with cirrhosis at baseline $(=13)$ were more likely to develop a clinical event on long-term follow-up (5/13) compared to noncirrhotics (6/45) ( $P=.01$, Figure 4F). The annual event-free survival rate in patients with cirrhosis vs noncirrhotic patients at year 1,5 and 10 was $83 \%$ vs $97 \%, 70 \%$ vs $91 \%$ and $35 \%$ vs $76 \%$. All patients who had undetectable HDV RNA results, that is had a MVR throughout follow-up $(n=6)$ remained free of hepatic events, irrespective whether they had cirrhosis or not. Neither negative HDV RNA at on-treatment week $24(P=.19)$, at end of treatment $(P=.28)$ or end of week 72 follow-up $(P=.09)$, nor a 2 log HDV RNA decline at on-treatment week $24(P=.77)$, at EOT $(P=.32)$ or at EO Week 72 follow-up $(P=.11)$ were associated with event-free survival. Besides, none of the patients who were HBsAg negative at post-treatment week 24 or during long-term follow-up developed a clinical endpoint in the not retreated group (Table S9).

Interestingly, lack of clinical endpoints was associated with HDV RNA negativity at any time point $(P<.01)$ and negative HDV RNA at the last available visit $(P=.02)$ (Table S9). Negative HDV RNA at any time during follow-up was also associated with clinical eventfree survival in Kaplan-Meier analysis $(P=.045)$ (Figure 4D) whereas HDV RNA at week 72 or at the last available visit were not associated with the development of liver-related endpoints in KaplanMeier analysis $(P=.18, P=.1)$ (Figure 4C,E).

\subsection{4 | Factors associated with clinical endpoints}

We next analysed factors which may have an association with an adverse outcome: the analyses were done separately for patients without IFN retreatment and for all patients including those with IFN retreatment. By univariate analysis, no association between the development of endpoints and the following baseline factors was found in the former group: age, platelets, ALT, AST, bilirubin, alkaline phosphatase, albumin and creatinine. Based on this model, gamma-GT $(P=.04)$ and cirrhosis $(P=.05)$ were associated with the development of endpoints (Table 2a). In the multivariate logistic regression based on the factors found in the univariate analysis, only gamma-GT was independently associated with the development of endpoints ( $P=.01$, odds ratio: $1.02, \mathrm{KI}: 1.0-1.03$ ).

In the regression analysis, based on all patients including those with IFN retreatment, age, platelets, ALT, AST, bilirubin, alkaline phosphatase, albumin and creatinine were not associated with endpoints. The association with gamma-GT $(P<.01$, odds ratio: 1.02 , $\mathrm{KI}: 1.0-1.03)$ and alkaline phosphatase $(P<.01$, odds ratio: $1.01, \mathrm{KI}$ : 1.0-1.02) was confirmed in this model. In the multivariate logistic regression, again only gamma-GT was independently associated with the endpoints ( $P=.03$, odds ratio: 1.02, Kl: 1.0-1.03) (Table 2b).

\section{4 | DISSCUSSION}

This study provides the first long-term follow-up study of a randomized controlled clinical trial in CHD. Several novel findings have emerged. Expectedly, long-term maintenance of HDV RNA undetectability was associated with a favourable clinical outcome and none of such patients developed a clinical endpoint supporting previous studies. ${ }^{17,20}$ However, of the original 52 patients who had received a PEG-IFN $\alpha-2 a$-based treatment regimen and had completed the one-year treatment regimen only six patients maintained this virological response. We had previously reported that relapse after post-treatment month 6 is possible up to 4 years after stopping treatment. The current study suggests that relapse may occur even after 5 years as one patient relapsed after 9 years after stopping treatment. It thus supports our previous report to not 


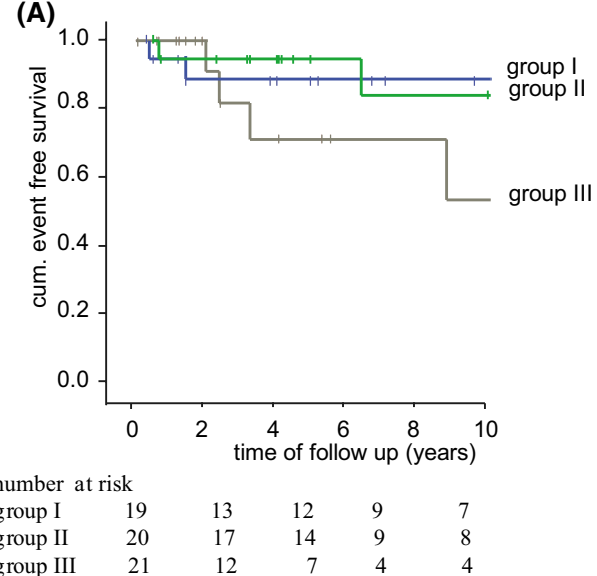

(C)

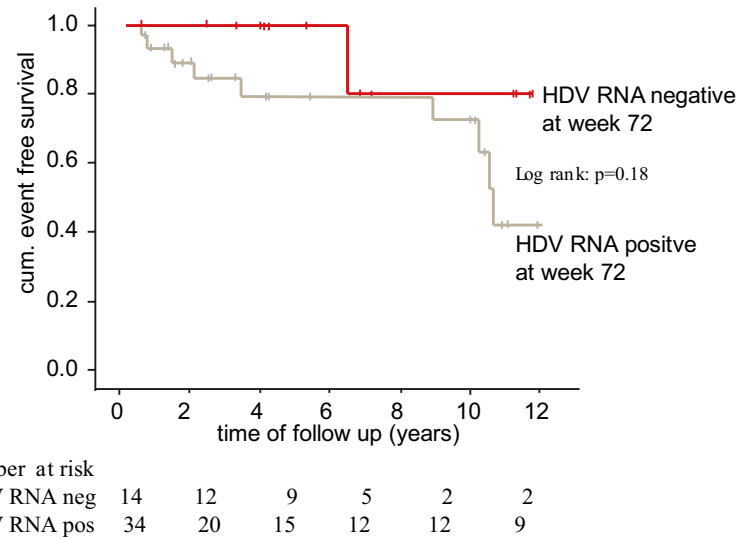

(B)

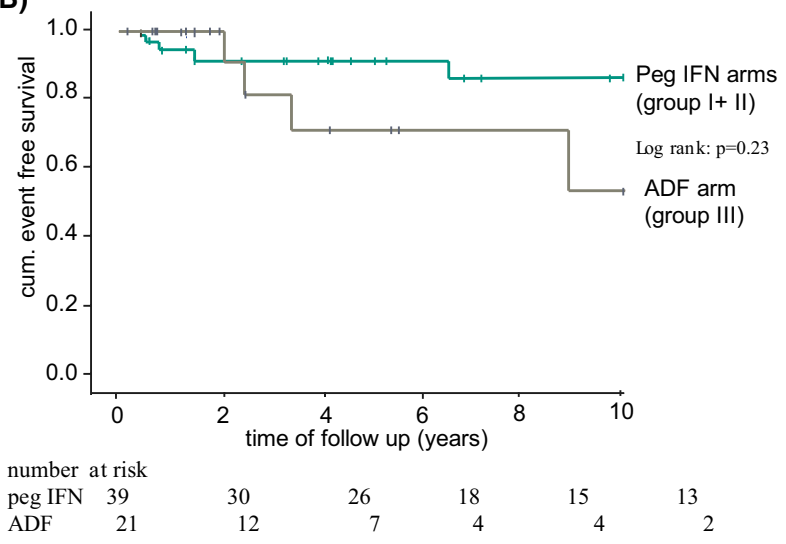

(D)

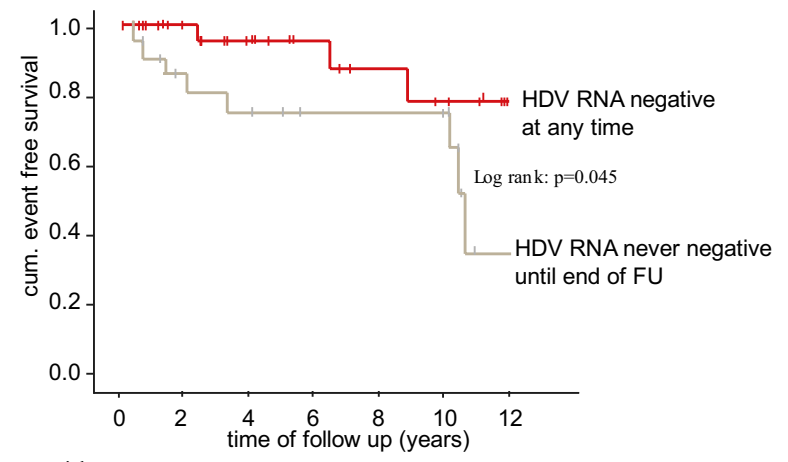

number at risk

$\begin{array}{lllrrrr}\text { HDV RNA neg } & 14 & 12 & 9 & 5 & 2 & 2\end{array}$

$\begin{array}{lllllll}\text { HDV RNA pos } & 34 & 20 & 15 & 12 & 12 & 9\end{array}$
(E)

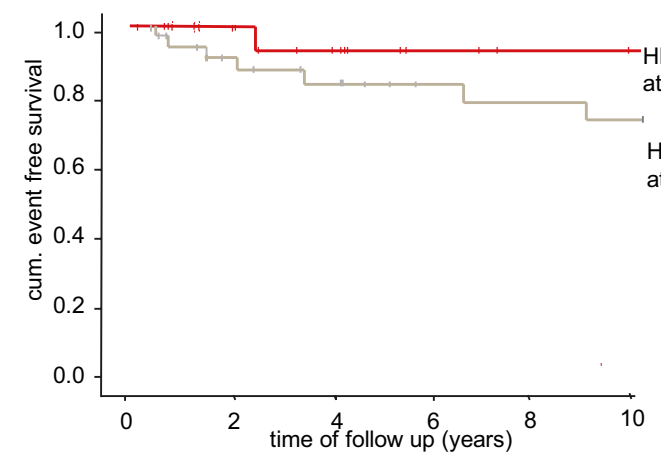

number at risk

HDV RNA neg

HDV RNA pos 38
$16 \quad 11$

11
22

HDV RNA negative at last visit

Log rank: $\mathrm{p}=0.1$

HDV RNA positive at last visit

FIGURE 4 A, Event-free survival in the three treatment groups. B, Event-free survival in patients treated with PEG-IFN $\alpha-2 a$ (group I + II) versus patients treated with ADV monotherapy. C, Event-free survival in patients with an end of follow-up response (w72) versus patients being HDV RNA positive at w72. D, Event-free survival of patients who reached HDV RNA negativity at any time point during treatment or long-term follow-up versus patients being all time HDV RNA positive. E, Event-free survival of patients with treatment response at their last available visit versus patients being HDV RNA positive. F, Event-free survival of patients with cirrhosis

extrapolating sustained virological response from the hepatitis $C$ literature to $\mathrm{CHD}^{19}$ It further suggests that even after years of a maintained virological response the wording 'cure from HDV' should better be avoided. Relapse was associated with high baseline HDV RNA. Previous studies have linked high baseline HDV RNA with
(F)

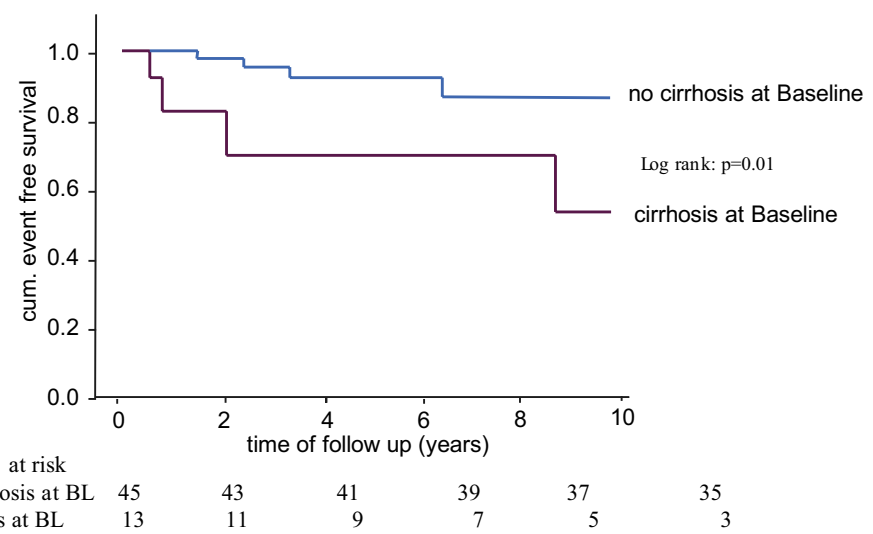


TABLE 2 (a) Association between the baseline characteristics of patients who developed a clinical endpoint compared to those who did not develop a clinical endpoint (excluding patients with retreatment). (b) Association between the baseline characteristics of patients who developed a clinical endpoint compared to those who did not develop a clinical endpoint (including patients with retreatment)

\begin{tabular}{|c|c|c|}
\hline Parameter & Significance (univariate) & $\begin{array}{l}\text { Significance } \\
\text { (multivariate) }^{*}\end{array}$ \\
\hline \multicolumn{3}{|l|}{ (a) } \\
\hline Age & $\begin{array}{l}P=.26 ; \text { OR } 1.03 ; 95 \% \mathrm{Cl} \\
0.98-1.1\end{array}$ & not significant \\
\hline Platelets & $\begin{array}{l}P=.47 ; \text { OR } 0.99 ; 95 \% \mathrm{Cl} \\
0.98-1.01\end{array}$ & not significant \\
\hline AST & $\begin{array}{l}P=.69 ; \text { OR } 0.99 ; 95 \% \mathrm{Cl} \\
0.98-1.01\end{array}$ & not significant \\
\hline ALT & $\begin{array}{l}P=.27 ; \text { OR } 0.99 ; 95 \% \mathrm{Cl} \\
0.98-1.00\end{array}$ & not significant \\
\hline Gamma-GT & $\begin{array}{l}P=.04 ; \text { OR } 1.01 ; 95 \% \mathrm{Cl} \\
1.0-1.02\end{array}$ & $\begin{array}{l}P=.01 ; \text { OR } 1.02 \\
95 \% \mathrm{Cl} 1.0-1.03\end{array}$ \\
\hline Bilirubin & $\begin{array}{l}P=.69 ; \text { OR } 0.68 ; 95 \% \mathrm{Cl} \\
0.095-4.8\end{array}$ & not significant \\
\hline $\begin{array}{l}\text { Alcaline } \\
\text { phosphatase }\end{array}$ & $\begin{array}{l}P=.13 ; \text { OR } 1.01 ; 95 \% \mathrm{Cl} \\
0.99-1.01\end{array}$ & not significant \\
\hline Albumin & $\begin{array}{l}P=.29 ; \text { OR } 0.41 ; 95 \% \mathrm{Cl} \\
0.074-2.22\end{array}$ & not significant \\
\hline Creatinine & $\begin{array}{l}P=.86 ; \text { OR } 0.66 ; 95 \% \mathrm{Cl} \\
0.01-56.8\end{array}$ & not significant \\
\hline Cirrhosis & $\begin{array}{l}P=.05 ; \text { OR } 4.06 ; 95 \% \mathrm{Cl} \\
1.00-16.64\end{array}$ & $\begin{array}{l}P=.29 ; \text { OR } 2.33 \\
95 \% \text { CI 0.48- } 11.31\end{array}$ \\
\hline \multicolumn{3}{|l|}{ (b) } \\
\hline Age & $\begin{array}{l}P=.19 ; \text { OR } 1.04 ; 95 \% \mathrm{Cl} \\
0.98-1.09\end{array}$ & not significant \\
\hline Platelets & $\begin{array}{l}P=.75 ; \text { OR } 0.99 ; 95 \% \mathrm{Cl} \\
0.99-1.01\end{array}$ & not significant \\
\hline AST & $\begin{array}{l}P=.62 ; \text { OR } 0.99 ; 95 \% \mathrm{Cl} \\
0.98-1.01\end{array}$ & not significant \\
\hline ALT & $\begin{array}{l}P=.17 ; \text { OR } 0.99 ; 95 \% \mathrm{Cl} \\
0.98-1.00\end{array}$ & not significant \\
\hline Gamma-GT & $\begin{array}{l}P<.01 ; \text { OR } 1.02 ; 95 \% \mathrm{Cl} \\
1.0-1.03\end{array}$ & $\begin{array}{l}P=.03 ; \text { OR 1.02; } \\
95 \% \text { Cl 1.00- } 1.03\end{array}$ \\
\hline Bilirubin & $\begin{array}{l}P=.47 ; \text { OR } 0.51 ; 95 \% \mathrm{Cl} \\
0.08-3.2\end{array}$ & not significant \\
\hline $\begin{array}{l}\text { Alcaline } \\
\text { phosphatase }\end{array}$ & $\begin{array}{l}P<.01 ; \text { OR } 1.01 ; 95 \% \mathrm{Cl} \\
1.00-1.02\end{array}$ & $\begin{array}{l}P=.10 ; \text { OR 1.01; } \\
95 \% \text { Cl 0.99- 1.02 }\end{array}$ \\
\hline Albumin & $\begin{array}{l}P=.31 ; \text { OR } 0.46 ; 95 \% \mathrm{Cl} \\
0.1-2.05\end{array}$ & not significant \\
\hline Creatinine & $\begin{array}{l}P=.96 ; \text { OR } 1.11 ; 95 \% \mathrm{Cl} \\
0.02-56.8\end{array}$ & not significant \\
\hline Cirrhosis & $\begin{array}{l}P=.07 ; \text { OR } 3.43 ; 95 \% \mathrm{Cl} \\
0.92-12.74\end{array}$ & not significant \\
\hline
\end{tabular}

of $\mathrm{HBsAg}$ clearance could be established. Baseline, on-treatment and EOT low HBsAg as well as baseline and on-treatment week $24 \mathrm{HDV}$ RNA levels predicted HBsAg clearance. Maintaining undetectable
HDV RNA for 2 years was the only independent factor for predicting $\mathrm{HBsAg}$ clearance in the Ankara cohort. ${ }^{17}$ However, this was a retrospective study, and on-treatment quantitative variables were not available. The current study's prospective nature enabled us to better elucidate predictors of $\mathrm{HBsAg}$ clearance. Quantitative $\mathrm{HBsAg}$ levels were better predictors than HDV RNA levels with AUROC above 0.80. For both baseline and EOT, qHBsAg AUROC was even 0.90 and above. Two previous studies dealt with $\mathrm{HBsAg}$ clearance after IFN treatment. In both studies, low baseline qHBsAg predicted HBsAg clearance in line with the current study. ${ }^{16,23,24}$ On-treatment $\mathrm{HBsAg}$ level of $<1000 \mathrm{IU} / \mathrm{L}$ predicted HBsAg clearance in one Italian study. ${ }^{25}$ Our data confirmed these results, although our calculated cut-offs predicting HBsAg at baseline, weeks 24 and 48 were slightly more than $1000 \mathrm{IU} / \mathrm{L}\left(3.5,3.2\right.$ and $3.2 \log _{10} \mathrm{IU} / \mathrm{mL}$, respectively).

Hepatic decompensation occurred in 10 of 60 patients (17\%) during long-term follow-up of patients without IFN retreatment. This figure is reminiscent of natural history data of patient cohorts with compensated cirrhosis due to HBV. ${ }^{26}$ Although our treatment cohort cannot be compared to a natural history cohort, these data nevertheless may suggest a milder course of delta hepatitis compared to the disease course in the 90 s as was also suggested in a cohort study from Italy. ${ }^{27}$ Since these studies are from areas where the HDV genotype is almost exclusively genotype $1,{ }^{8}$ the current milder course of delta hepatitis may be explained by a slower turnover of $\mathrm{HDV}$ in the community leading to decreased virulence of HDV. ${ }^{28}$

Lack of clinical events was found to be associated with HDV RNA negativity at any time $(P<.01)$ and negative HDV RNA at the last available visit $(P=.02)$. However, more than 2 log drop at EOT or post-treatment follow-up as well as end of treatment undetectable HDV RNA was not associated with event-free survival longterm. Still, numbers are too small to rule out that the respective HDV RNA declines translate into better long-term outcomes. A more than 2 log drop of HDV RNA at end of treatment compared to baseline has recently been suggested by a group of experts as a surrogate marker of initial treatment efficacy in the wake of phase 3 studies with new compounds. ${ }^{29}$ Supporting such a recommendation was normalization of elevated alanine aminotransferase levels in the majority of patients treated with new compounds. The data presented here suggest that this virological and its consequent biochemical and anti-inflammatory response needs to be maintained by various means such as adding a compound with a different mode of action, extension of treatment duration or doing repeated courses of treatment. ${ }^{29}$ It is interesting, however, that HDV RNA negativity at any time point and negative HDV RNA at the last available visit were associated with a favourable disease course. Since these data are off-treatment long-term follow-up data and sharp increases of viraemia may be expected in the early months after treatment discontinuation but not years thereafter, it suggests that low levels of HDV RNA throughout follow-up may be associated with favourable disease course.

Another novel finding of this study was that 'late response' to PEG-IFN $\alpha-2 a$ is possible as 7 out of 22 patients with undetectable HDV RNA at the last available visit were patients with detectable 
HDV RNA at EOT and post-treatment week 24. These patients became HDV RNA negative between follow-up years 1-10 and had a favourable long-term disease course. The mechanism of this late response is unknown. The two modes of action, the immune stimulant and antiviral effects of IFN should be taken into account. For the former, host-induced on- and off-treatment beneficial flares reported for hepatitis $\mathrm{B}^{30,31}$ have also been described for HDV. ${ }^{32}$ In the current study data to support such host-induced off-treatment flares are lacking but participating centres were not specifically asked for the presence of flares. In the context of antiviral efficacy, in vitro studies may be in line with a late response. Those studies suggest that interferons may interfere with entrance of HDV into hepatocytes and do not affect intracellular HDV infection. ${ }^{33,34}$ Human pharmacokinetic studies support these in vitro studies, and a much longer delay is observed before PEG-IFN $\alpha$ has an effect on HDV RNA compared to the effect of PEG-IFN $\alpha$ on HCV RNA or HBV DNA. ${ }^{35}$

Patients with cirrhosis at baseline predicted the development of a clinical endpoint by univariate but not by multivariate analysis. However, high gamma-GT was an independent factor of clinical endpoint development by multivariate analysis. These data are similar to previous retrospective data. ${ }^{16}$ It is likely that high gamma-GT levels can be a reflection of more advanced liver disease. High GGT may be observed in diverse clinical conditions including nonliver-related ones. Among liver disease, the aetiology of elevated GGT may be due to alcoholic/ nonalcoholic liver disease or cholestatic liver disease but may also be secondary to chronic viral hepatitis. In the latter ones, this has been, in general, associated with long-standing and significant liver disease. ${ }^{36}$ The contribution of alcohol intake on elevated GGT levels is also possible. Excess alcohol use (>40 g/day for men, $>20 \mathrm{~g} /$ day for women) was an exclusion criteria in the HIDIT-1 study. Patients were also told to avoid alcohol intake once they had been recruited into the study. However, alcohol intake cannot be ruled with certainty. In addition, BMI, HOMA score and thus metabolic syndrome were not specifically assessed at study entry and nonalcoholic steatohepatitis may have been a confounding factor for advanced liver disease. Further, GGT may also be induced by drugs. Finally, as mentioned above elevated GGT may also be due to nonliver-related causes. ${ }^{37}$ However, despite the low specificity of elevated GGT for liver disease, currently elevated serum GGT is considered as one of the best predictors of significant liver disease and liver-related mortality. ${ }^{37}$ Hence, high GGT may be associated with clinical outcomes indirectly as a marker of significant liver disease ${ }^{36,37}$ but also directly through its pro-oxidant effects. ${ }^{38}$

Several shortcomings of the study need mentioning, however. In the study, total bilirubin was measured only. We therefore cannot provide data on direct bilirubin. Further, data after post-treatment week 24 were retrospectively collected. This has affected data collection and long-term follow-up data are based on 3 or more HDV RNA determinations in 18 out of 60 patients. For long-term virological response, assessment was relied on local laboratory in-house PCR assessments with divergent sensitivities. However, HDV RNA assay sensitivities may differ even among reference laboratories ${ }^{39}$ Nucles(t)ide analog (NA) use was not uniform among centres, and this was not specifically asked for. However, the effect of NA use on clinical outcomes should be negligible as has recently been shown in CHD patients on long-term NA therapy. ${ }^{40}$

In conclusion, this 10-year FU study of the HIDIT-I study suggests that a maintained virological response is associated with a favourable clinical outcome and that baseline cirrhosis and gamma-GT are risk factors for clinical endpoint development. However, our data also suggest that there may be a group of patients who benefit from low levels of HDV RNA off-treatment. The optimal response to treatment, and $\mathrm{HBsAg}$ clearance, increases with longterm follow-up and is observed in patients with virological response. Baseline and on-treatment quantitative $\mathrm{HBsAg}$ levels and on-treatment HDV RNA levels may also be important as they could predict $\mathrm{HBsAg}$ clearance.

\section{CONFLICT OF INTEREST}

No conflict.

\section{ORCID}

Anika Wranke iD https://orcid.org/0000-0002-7662-7307

Markus Cornberg (iD https://orcid.org/0000-0002-9141-8001

Cihan Yurdaydin iD https://orcid.org/0000-0002-5419-7158

\section{REFERENCES}

1. Hughes SA, Wedemeyer H, Harrison PM. Hepatitis delta virus. Lancet. 2011;378:73-85.

2. Mederacke I, Bremer B, Heidrich B, et al. Establishment of a novel quantitative hepatitis D virus (HDV) RNA assay using the Cobas TaqMan platform to study HDV RNA kinetics. J Clin Microbiol. 2010;48:2022-2029.

3. Yurdaydin $\mathrm{C}$, Idilman R, Bozkaya $\mathrm{H}$, et al. Natural history and treatment of chronic delta hepatitis. J Viral Hepat. 2010;17:749-756.

4. Schaper M, Rodriguez-Frias F, Jardi R, et al. Quantitative longitudinal evaluations of hepatitis delta virus RNA and hepatitis B virus DNA shows a dynamic, complex replicative profile in chronic hepatitis B and D. J Hepatol. 2010;52:658-664.

5. Zachou K, Yurdaydin C, Drebber U, et al. Quantitative HBsAg and HDV-RNA levels in chronic delta hepatitis. Liver Int. 2010;30:430-437.

6. Wranke A, Pinheiro Borzacov LM, Parana R, et al. Clinical and virological heterogeneity of hepatitis delta in different regions worldwide: the Hepatitis Delta International Network (HDIN). Liver Int. 2018;38:842-850

7. Le Gal F, Brichler S, Drugan T, et al. Genetic diversity and worldwide distribution of the deltavirus genus: a study of 2,152 clinical strains. Hepatology. 2017;66:1826-1841.

8. Shakil AO, Hadziyannis S, Hoofnagle JH, et al. Geographic distribution and genetic variability of hepatitis delta virus genotype I. Virology. 1997;234:160-167.

9. Organization WH. Global Hepatitis Reoprt 2017: World Health Organization; 2017.

10. The Polaris Observatory Collaborators. Global prevalence, treatment, and prevention of hepatitis B virus infection in 2016: a modelling study. Lancet Gastroenterol Hepatol. 2018;3(6):383-403.

11. Stockdale AJ, Kreuels B, Henrion MRY, et al. Hepatitis D prevalence: problems with extrapolation to global population estimates. Gut. 2020;69(2):396-397. 
12. Wedemeyer H, Negro F. Devil hepatitis D: an orphan disease or largely underdiagnosed? Gut. 2019;68(3):381-382.

13. Chen HY, Shen DT, Ji DZ, et al. Prevalence and burden of hepatitis $D$ virus infection in the global population: a systematic review and meta-analysis. Gut. 2019;68(3):512-521.

14. Castelnau C, Le Gal F, Ripault MP, et al. Efficacy of peginterferon alpha-2b in chronic hepatitis delta: Relevance of quantitative RTPCR for follow-up. Hepatology. 2006;44:728-735.

15. Wedemeyer H, Yurdaydin C, Dalekos GN, et al. Peginterferon plus adefovir versus either drug alone for hepatitis delta. N Engl J Med. 2011;364:322-331.

16. Gheorghe L, Csiki IE, lacob S, et al. Hepatitis delta virus infection in romania: prevalence and risk factors. J Gastrointestin Liver Dis. 2015;24:413-421.

17. Yurdaydin C, Keskin O, Kalkan C, et al. Interferon treatment duration in patients with chronic delta hepatitis and its effect on the natural course of the disease. J Infect Dis. 2018;217(8):1184-1192.

18. Manesis EK, Vourli G, Dalekos G, et al. Prevalence and clinical course of hepatitis delta infection in Greece: a 13-year prospective study. J Hepatol. 2013;59:949-956.

19. Heidrich B, Yurdaydin C, Kabacam G, et al. Late HDV RNA relapse after peginterferon alpha-based therapy of chronic hepatitis delta. Hepatology. 2014;60:87-97.

20. Wranke A, Serrano BC, Heidrich B, et al. Antiviral treatment and liver-related complications in hepatitis delta. Hepatology. 2017;65:414-425.

21. Wranke A, Deterding K, Heidrich B, et al. Comparison of hepatitis delta and HBV monoinfection in a real-world setting. $J$ Viral Hepatitis. 2018;25:89.

22. Wursthorn K, Jaroszewicz J, Zacher BJ, et al. Correlation between the Elecsys HBsAg II assay and the Architect assay for the quantification of hepatitis B surface antigen ( $\mathrm{HBsAg}$ ) in the serum. J Clin Virol. 2011:50:292-296.

23. Romeo R, Del Ninno E, Rumi M, et al. A 28-year study of the course of hepatitis delta infection: a risk factor for cirrhosis and hepatocellular carcinoma. Gastroenterology. 2009;136:1629-1638.

24. Romeo R, Foglieni B, Casazza G, et al. High serum levels of HDV RNA are predictors of cirrhosis and liver cancer in patients with chronic hepatitis delta. PLoS One. 2014;9:e92062.

25. Niro GA, Smedile A, Fontana R, et al. HBsAg kinetics in chronic hepatitis $D$ during interferon therapy: on-treatment prediction of response. Aliment Pharmacol Ther. 2016;44:620-628.

26. de Jongh FE, Janssen HL, de Man RA, et al. Survival and prognostic indicators in hepatitis B surface antigen-positive cirrhosis of the liver. Gastroenterology. 1992;103:1630-1635.

27. Rosina F, Conoscitore $\mathrm{P}$, Cuppone R, et al. Changing pattern of chronic hepatitis D in Southern Europe. Gastroenterology. 1999;117:161-166.

28. Rizzetto M, Smedile A. Pegylated interferon therapy of chronic hepatitis D: in need of revision. Hepatology. 2015;61:1109-1111.
29. Yurdaydin C, Abbas Z, Buti M, et al. Treating chronic hepatitis delta: The need for surrogate markers of treatment efficacy. J Hepatol. 2019;70:1008-1015.

30. Flink HJ, Sprengers D, Hansen BE, et al. Flares in chronic hepatitis $\mathrm{B}$ patients induced by the host or the virus? Relation to treatment response during Peg-interferon \{alpha\}-2b therapy. Gut. 2005;54:1604-1609.

31. Papatheodoridis G, Vlachogiannakos I, Cholongitas E, et al. Discontinuation of oral antivirals in chronic hepatitis B: a systematic review. Hepatology. 2016;63:1481-1492.

32. Hardtke S. Frequency, severity and impact of Peg-IFNa-associated flares in HDV-Resultas from the HIDIT-II study. J Hepatol. 2019;70:e466

33. Zhang Z, Filzmayer C, Ni Y, et al. Hepatitis D virus replication is sensed by MDA5 and induces IFN-beta/lambda responses in hepatocytes. J Hepatol. 2018;69:25-35.

34. Han Z, Nogusa S, Nicolas E, et al. Interferon impedes an early step of hepatitis delta virus infection. PLoS One. 2011;6:e22415.

35. Guedj J, Rotman Y, Cotler SJ, et al. Understanding early serum hepatitis $D$ virus and hepatitis B surface antigen kinetics during pegylated interferon-alpha therapy via mathematical modeling. Hepatology. 2014:60:1902-1910.

36. Whitfield JB. Gamma glutamyl transferase. Crit Rev Clin Labor Sci. 2001;38:263-355.

37. Newsome PN, Cramb R, Davison SM, et al. Guidelines on the management of abnormal liver blood tests. Gut. 2018;67:6-19.

38. Koenig G, Seneff S. Gamma-glutamyl transferase: a predictive biomarker of cellular antioxidant inadequacy and disease risk. Dis Markers. 2015;2015:1-18.

39. Le Gal F, Brichler S, Sahli R, et al. First international external quality assessment for hepatitis delta virus RNA quantification in plasma. Hepatology. 2016;64:1483-1494.

40. Brancaccio G, Fasano M, Grossi A, et al. Clinical outcomes in patients with hepatitis $D$, cirrhosis and persistent hepatitis $B$ virus replication, and receiving long-term tenofovir or entecavir. Aliment Pharmacol Ther. 2019;49:1071-1076.

\section{SUPPORTING INFORMATION}

Additional supporting information may be found online in the Supporting Information section.

How to cite this article: Wranke A, Hardtke S, Heidrich B, et al. Ten-year follow-up of a randomized controlled clinical trial in chronic hepatitis delta. J Viral Hepat. 2020;00:1-10. https://doi.org/10.1111/jvh.13366 